\title{
On liquid phase hydrates formation in pipelines in the course of gas non-stationary flow
}

\author{
Teimuraz Davitashvili ${ }^{{ }^{*}}$ \\ ${ }^{1}$ Iv. Javakhishvili Tbilisi State University, I. Vekua Institute of Applied Mathematics, 2 University St, \\ 0186, Tbilisi, Georgia
}

\begin{abstract}
Nowadays, when the emphasis is on alternative means of energy, natural gas is still used as an efficient and convenient fuel both in the home (for heating buildings and water, cooking, drying and lighting) and in industry together with electricity. In industrial terms, gas is one of the main sources of electricity generation in both developed and developing countries. Pipelines are the most popular means of transporting natural gas domestically and internationally. The main reasons for the constipation of gas pipelines are the formation of hydrates, freezing of water plugs, pollution, etc. It is an urgent task to take timely measures against the formation of hydrates in the pipeline. To stop gas hydrate formation in gas transporting pipelines, from existing methods the mathematical modelling with hydrodynamic method is more acceptable. In this paper the problem of prediction of possible points of hydrates origin in the main pipelines taking into consideration gas non-stationary flow and heat exchange with medium is studied. For solving the problem the system of partial differential equations governing gas non-stationary flow in main gas pipeline is investigated. The problem solution for gas adiabatic flow is presented.
\end{abstract}

\section{Introduction}

Pipelines represent the most popular means for natural gas conveyance [1-5]. Hydrogen, thanks to its high energy capacity and no emission of greenhouse gases, is of great importance in industry $[6,7]$. Indeed, its combination with oxygen creates energy and water without any emission output, which makes hydrogen an attractive source of energy for the high-tech industry [6]. Unfortunately hydrogen has some problems with its storage and transportation too [6]. In order to deliver more energetic and low-carbon gas product to consumers, in the last century was proposed an idea to mix hydrogen with natural gas and transport by existing gas pipeline networks $[8,9]$. Hydrogen and methane mixture is very flammable and if the hydrogen content is high then can be set off an explosion and flame may be almost invisible. But it should be noted that at such transportation of blended hydrogen and natural gas in the pipeline networks, besides of increasing the risks of transportation (it is necessary to determine the safe values of volumetric concentrations of hydrogen in the mixture (5-15\%)) and mass consumption (in end-use devices), there is high probability of increasing the risk of hydrate formation in pipelines $[6,7]$.

*Corresponding author: teimuraz.davitashvili@tsu.ge 
As known, the European Union (EU) considers transport corridor Europe-CaucasusAsia (TRACECA) as mechanism of inter-state and inter-regional collaborations and the guarantee of peace and stability in Caucasus $[3,10]$. Caspian Sea region has abundant oil and gas reserves but a limited accessible market for the products. Today TRACECA is considered as a corridor, which is complementing other existing routes and EU considers Georgia as a partner in the development of the transport networks between the Black Sea and Central Asia because of its geopolitical position [10]. At present there are already functioning the following routs of natural gas transportation via the territory of Georgia: the South Caucasus (Baku (Azerbaijan) - Tbilisi (Georgia) - Erzurum (Turkey)) gas pipeline; Mozdok (Russia) - Tbilisi - Yerevan(Armenia) gas pipeline; Chmi(Russia) - Tbilisi Saguramo (Georgia) gas pipeline [3,10]. It is quite possible that hydrogen in combination with natural gas, as a new form of energy, may penetrate into the Caucasus existing natural gas supply system, as the conventional type energy [10,11].

As practice shows while transportation of natural gas by pipelines pressure and temperature changes cause formation of a liquid phase owing to partial condensation of the gaseous medium [12-14]. Hydrates of natural gases represent crystalline substances made of by molecules of hydrocarbons and water. Normally they have crystal structure and may be considered as hardened solute. Hydrates of natural gases decompose when tension of water vapor is less than partial pressure of investigated gas. Growing in main gas pipelines they can partly or totally occlude pipe line $[13,14]$. Hydrates of natural gases look like on a pressed wet snow and composition of natural gases hydrates are expressed by the following formulas: $\mathrm{CH}_{4} \cdot 6 \mathrm{H}_{2} \mathrm{O} ; \mathrm{C}_{2} \mathrm{H}_{6} \cdot 6 \mathrm{H}_{2} \mathrm{O} ; \mathrm{C}_{3} \mathrm{H}_{8} \cdot 17 \mathrm{H}_{2} \mathrm{O}$ etc [4, 12]. There are many scientific articles denoted to the problem of prediction of possible points of hydrates origin in the main pipelines $[13,15,16]$. There are several methods for avoiding gas hydrate problems, but generally modern methods for prevention of hydrate formation are based on the following techniques: injection of thermodynamic inhibitors, use of kinetic hydrate inhibitors to sufficiently delay hydrate nucleation/intensification, and maintain pipeline operating conditions outside the hydrate stability zone by insulation, heating and controlling pressure [17-21]. However, the above techniques may not be economical and practical. From existing methods the mathematical modelling with hydrodynamic method is more acceptable as it is very cheap and reliable and has high sensitive and operative features $[13,14,22]$. The main objective of the paper is to find a convenient method for prediction of possible area of hydrates origin in the main pipelines taking into consideration gas non-stationary flow based on the system of partial differential equations governing gas non-stationary flow.

\section{Problem formulation}

Main reasons of pipeline constipation (emergency shutdown) are generation of hydrates, freezing of water slugs, contamination and so on. To take timely steps against the hydrates generation, it is necessary to better know the gas humidity, pressure and temperature distributions in pipeline. It is well-known that favorable conditions for hydrates generation in the main pipeline, is a placement where dew point (varying according to pressure and humidity) occurs. Indeed dew point is the value of temperature, below which water droplets begin to condense and dew can forms. Generally, the value of dew point is obtained at intersection of equilibrium temperature plot (of hydrate generation) and corresponding curve of gas temperature. As known equilibrium temperature can be calculated by the following formula [1]:

$$
T_{h d r}=s \lg P-u,
$$

where $T_{h d r}$ - the equilibrium temperature of gas ; $P$ - the pressure ; $s$ and $u$ - the constants, 
which can be defined from experiments taking into account the gas with mixture particular content and specific weight (special graphs exist) [1, 13, 14, 22]. Therefore, for definition the possible area of hydrate generation first of all it is necessary to find out pressure and temperature distribution in the pipeline. Such kind problem, under the condition of gas stationary flow, have been discussed and examined by a number of investigators [1, 13-22]. In our study an attempt is made to examine the role of gas non-stationary flow with accounting heat exchange with medium for definition hydrates possible generation point in the pipeline.

\subsection{Mathematical statement of the problem}

It is well-known that non-stationary, non-isothermal flow of gas in the main pipeline is described by the following system of equations $[1,22,23]$ :

$$
\begin{gathered}
\frac{\partial \omega}{\partial t}=-\frac{1}{\rho_{0}} \frac{\partial P}{\partial x}+v_{0} \cdot \frac{1}{r} \frac{\partial}{\partial r}\left(r \frac{\partial \omega}{\partial r}\right) \\
\frac{\partial P}{\partial t}=\rho_{0} C_{P}\left(1-\frac{C_{P}}{C_{v}}\right) \frac{\partial T}{\partial t}-\frac{\rho_{0} C^{2} C_{v}}{C_{P}} \cdot \frac{\partial \omega}{\partial x} \\
\frac{\partial \rho}{\partial t}=-\rho_{0} \frac{\partial \omega}{\partial r} \\
\frac{\partial T}{\partial t}=-\frac{1}{\rho C_{P}} \frac{\partial P}{\partial t}+a \cdot \frac{1}{r} \frac{\partial}{\partial r}\left(r \frac{\partial T}{\partial r}\right)
\end{gathered}
$$

where $\omega(x, r, t)$ - gas flow velocity; $P(x, r, t)$ - gas pressure ; $T(x, r, t)$ - gas temperature (absolute); $\rho(x, r, t)$ - density; $\rho_{0}$ - is gas density in normal conditions ; $\alpha$-gas heat conduction coefficient; $v_{0}$-viscosity in normal conditions; $C_{P}$ - gas heat capacity under the constant pressure ; $C_{v}$ - gas heat capacity under the constant volume ; $C$ speed of sound propagation in gas.

After some simple transformations system (2)-(5) takes the following form:

$$
\begin{gathered}
\frac{\partial \omega}{\partial t}=-\frac{1}{\rho_{0}} \frac{\partial P}{\partial x}+v_{0} \cdot \frac{1}{z} \frac{\partial}{\partial r}\left(r \frac{\partial \omega}{\partial r}\right) ; \\
\frac{\partial T}{\partial t}=-\frac{\rho_{0} C^{2} C_{v}^{2}}{\rho C_{P}^{2} C_{v}-\rho_{0} C_{P}^{2} C_{v}+\rho_{0} C_{P}^{3}} \frac{\partial \omega}{\partial x}+\frac{a C_{v} \rho}{\rho C_{v}-\rho_{0} C_{v}+C_{P} \rho_{0}} \frac{1}{r} \frac{\partial}{\partial r}\left(r \frac{\partial T}{\partial r}\right) \\
\frac{\partial \rho}{\partial t}=-\rho_{0} \frac{\partial \omega}{\partial x} \\
\frac{\partial P}{\partial t}=-\frac{\rho \rho_{0} C^{2} C_{v}^{2}}{\rho C_{P} C_{v}-\rho_{0} C_{P} C_{v}+\rho_{0} C_{P}^{2}} \frac{\partial \omega}{\partial x}+\frac{\rho a \rho_{0} C_{P}\left(C_{v}-C_{P}\right)}{\rho C_{v}-\rho_{0}\left(C_{v}-C_{P}\right)} \frac{1}{r} \frac{\partial}{\partial r}\left(r \frac{\partial T}{\partial r}\right)
\end{gathered}
$$

The system of equations (6)-(9) is solved with the following initial: 


$$
\begin{aligned}
& \omega(x, r, 0)=\omega_{o}(x, r), P(x, r, 0)=P_{0}(x, r) ; \\
& T(x, r, 0)=T_{0}(x, r), \rho(x, r, 0)=\rho_{0}(x, r),
\end{aligned}
$$

and boundary conditions:

$$
\begin{gathered}
\omega(0, r, t)=\omega_{o}(0, r, t), \omega(x, R, t)=0 ; \\
P(0, r, t)=P_{o}(0, r, t), T(x, R, t)=T_{0}(x, R, t), \\
\frac{\partial \omega}{\partial r}=0, \text { and } \frac{\partial T}{\partial r}=0 \quad \text { at }, r=0 ; \\
\frac{\partial T}{\partial r}=a\left(T_{b a c}-T(x, R, t)\right) \quad a t, r=R,
\end{gathered}
$$

with the additional constraints: $0 \leq x<L, 0 \leq r<R, t \geq 0$. Where $T_{b a c}-$ a temperature of background, $R$ - is a radius of pipeline cross-section; $L$ - length of the main pipeline.

Using solutions of problem (6)-(12) $P(x, r, t)$ and $T(x, r, t)$, on the basis of equality (1), we are constructing the following an inequality: $T(x, r, t)<s \lg P(x, r, t)-u$. Where for defining numerical values of parameters $s$ and $u$ we use the relation between equilibrium temperature $T_{h d r}$, which is different for various gases [14,22]. Based on these graphs for natural gas points $\left(10^{\circ} \mathrm{C} ; 6 \cdot 10^{6} \mathrm{~Pa}\right)$ and $20^{\circ} \mathrm{C} ;\left(2.5 \cdot 10^{7} \mathrm{~Pa}\right)$ lie on one graph and we obtained the following linear system of equations:

$$
\begin{gathered}
s \lg \left(6 \cdot 10^{6}\right)-u=283 \\
s \lg \left(2.5 \cdot 10^{7}\right)-u=293,
\end{gathered}
$$

from where $s=16.1 ; u=-173.64$, and finaly we have:

$$
T_{h d r}=16.1 \cdot \lg P+173.6377 .
$$

\section{Solution of the problem}

Hydration requires substances such as methane, carbon dioxide, water, high pressure, low temperature in the pipeline. Studies in the field of hydrate formation have shown that water molecules, combining hydrogen with its molecules, create holes in which quest molecules will be captured, and by creating a Van der Waals connection with water molecules, hydrate crystals will form [23]. Generally, the system (6)-(9) with initial-boundary conditions (10)-(12) can be effectively solved by numerical methods using explicit or implicit schemes [24]. However, in fact, it is not always possible to obtain real data for initial and boundary conditions, characterized distribution of velocity of gas flow, gas pressure, temperature and gas density in the pipeline.

\subsection{Gas flow is adiabatic}

That is way let us considering the case when gas flow is adiabatic. In this case we have [14, 22]:

$$
\frac{P}{\rho}=C^{2},
$$

where $C$ - sound velocity in gas medium. From (13) we have : 


$$
\frac{\partial \rho}{\partial t}=\frac{1}{C^{2}} \cdot \frac{\partial P}{\partial t}
$$

and equations (3) and (4) take the form:

$$
\begin{gathered}
\frac{\partial T}{\partial t}=-\frac{C^{2}}{C_{P}} \cdot \frac{1}{P} \cdot \frac{\partial P}{\partial t}+a \cdot \frac{1}{r} \cdot \frac{\partial}{\partial r}\left(r \frac{\partial T}{\partial r}\right) ; \\
\frac{\partial \rho}{\partial t}=-\frac{C^{2}}{\rho_{0}} \cdot \frac{\partial \omega}{\partial x},
\end{gathered}
$$

ubstitution of equation (15) in the equation (14) gives:

$$
\frac{\partial T}{\partial t}=-\frac{C^{4} \rho_{0}}{C_{P} \rho} \cdot \frac{1}{P} \cdot \frac{\partial \omega}{\partial x}+a \cdot \frac{1}{r} \cdot \frac{\partial}{\partial r}\left(r \frac{\partial T}{\partial r}\right) .
$$

If instead of equation (5), we consider equation (13) than as a result, we obtain the following system of equations:

$$
\begin{gathered}
\frac{\partial \omega}{\partial t}=-\frac{1}{\rho_{0}} \frac{\partial P}{\partial x}+v_{0} \cdot \frac{1}{z} \frac{\partial}{\partial r}\left(r \frac{\partial \omega}{\partial r}\right) \\
\frac{\partial T}{\partial t}=-\frac{C^{4} \rho_{0}}{C_{P}} \cdot \frac{1}{P} \cdot \frac{\partial \omega}{\partial x}+a \cdot \frac{1}{r} \cdot \frac{\partial}{\partial r}\left(r \frac{\partial T}{\partial r}\right) ; \\
\frac{\partial P}{\partial t}=-C^{2} \rho_{0} \cdot \frac{\partial \omega}{\partial x} \\
\rho=\frac{1}{C^{2}} P .
\end{gathered}
$$

If we neglect the change of speed along the radius, equation (16) will take the form:

$$
\frac{\partial \omega}{\partial t}=-\frac{1}{\rho_{0}} \cdot \frac{\partial P}{\partial x}
$$

Equations (19), (16) and (17) create system with regards to functions $P, \omega$ and $T$ :

$$
\begin{gathered}
\frac{\partial P}{\partial t}=-C^{2} \rho_{0} \cdot \frac{\partial \omega}{\partial x} \\
\frac{\partial P}{\partial t}=-C^{2} \rho_{0} \cdot \frac{\partial \omega}{\partial x} ; \\
\frac{\partial \omega}{\partial t}=-\frac{1}{\rho_{0}} \cdot \frac{\partial P}{\partial x} ; \\
\frac{\partial T}{\partial t}=-\frac{C^{4} \rho_{0}}{C_{P}} \cdot \frac{1}{P} \cdot \frac{\partial \omega}{\partial x}+a \cdot \frac{1}{r} \cdot \frac{\partial}{\partial r}\left(r \frac{\partial T}{\partial r}\right) ;
\end{gathered}
$$




$$
\frac{P}{\rho}=C^{2} .
$$

Equations (21) and (22) of the system represent a system of two-variable equations with regards to functions $P(x, t)$ and $\omega(x, t)$, and from them we obtain the following hyperbolic equation:

$$
\frac{\partial^{2} P}{\partial t^{2}}=C^{2} \frac{\partial^{2} P}{\partial x^{2}}
$$

The equation (25) is solved by the following initial:

$$
\begin{gathered}
P(x, 0)=\phi(x), \quad 0 \leq x \leq L ; \\
\left.\frac{\partial P}{\partial t}\right|_{t=0}=\psi(x), \quad 0 \leq x \leq L,
\end{gathered}
$$

and boundary conditions:

$$
\begin{gathered}
P(0, t)=\mu_{1}(t), \quad t \geq 0 ; \\
P(L, t)=\mu_{2}(L, t), \quad t \geq 0 .
\end{gathered}
$$

The solution of the problem (25)-(29) has the following form [3]:

$$
P(x, r, t)=P_{1}(x, t)+P_{2}(x, t)+P_{3}(x, t),
$$

where:

$$
\begin{gathered}
P_{1}(x, r, t)=\frac{\phi(x+c t)+\phi(x-c t)}{2}+\frac{1}{2 c} \int_{x-c t}^{x+c t} \psi(\alpha) d \alpha ; \\
P_{2}(x, r, t)=\sum_{n=0}^{\infty} \mu_{1}\left(t-\frac{2 n L}{c}-\frac{x}{c}\right)-\sum_{n=1}^{\infty} \bar{\mu}_{1}\left(t-\frac{2 n L}{c}+\frac{x}{c}\right) ; \\
P_{3}(x, r, t)=\sum_{n=0}^{\infty} \bar{\mu}_{2}\left(t-\frac{(2 n+1) L}{c}+\frac{x}{c}\right)-\sum_{n=0}^{\infty} \bar{\mu}_{2}\left(t-\frac{(2 n+1) L}{c}-\frac{x}{c}\right),
\end{gathered}
$$

where:

$$
\bar{\mu}_{1}(t)=\left\{\begin{array}{ll}
\mu_{1}(t), & 0<t<\frac{2 L}{c}, \\
0, & t<0 ;
\end{array} \quad \bar{\mu}_{2}(t)= \begin{cases}\mu_{2}(t), & 0<t<\frac{2 L}{c} . \\
0, & t<0 ;\end{cases}\right.
$$

After finding the function $P(x, r, t)$, we can find the functions $\omega(x, r, t), T(x, r, t)$, $\rho(x, r, t)$ from equations (22), (23), (24) with the appropriate initial and boundary conditions. Namely, after finding the function $P(x, r, t)$, the function $\omega(x, r, t)$ can be found from equations (21) and (22). To predict the generation of condensate, it is sufficient to know the functions $P(x, r, t)$ and $T(x, r, t)$. For this reason from $(21)$, using the found $P$, we can define $\frac{\partial \omega}{\partial x}$ and from the equation (23) we will find the function $T(x, r, t)$. 


\section{Results and discussion}

Before going over to execute numerical calculations based on system of equations (6)-(9) with initial (10) and boundary (11)-(12) conditions, the simple case of above described methodology will be considered. A test will be useful for further numerical calculations (for initial conditions) and to compare the method of solution described in this study with the results presented by Bobrovski and Yufin.

\subsection{Analytical solution}

We have found an analytical, one dimensional solution of the equation (25), satisfying initial (26)-(27) and boundary conditions (28)-(29). The solution has the following form:

$$
P(x, t)=\frac{P_{0}}{2}\left[\exp \left(\frac{-\lambda x}{L}-\frac{c^{2} t}{L t_{0}}\right)+\exp \left(\frac{-\lambda x}{L}+\frac{c^{2} t}{L t_{0}}\right)\right],
$$

where $P_{0}$-value of pressure at the entrance of the pipeline; value of $\lambda$-defined from initial (26)-(27) and boundary conditions (28)-(29) and $\lambda=\ln \frac{P_{0}}{P_{1}}, P_{1}-$ value of pressure at the end point of the pipeline.

Equation (14) for one dimensional case has the following form:

$$
\frac{\partial T}{\partial t}=-\frac{C^{2}}{C_{P}} \cdot \frac{1}{P} \cdot \frac{\partial P}{\partial t}
$$

Temporal integration of (31) gives:

$$
T(x, t)=T(x, 0)-\frac{c^{2}}{c_{p}} \ln \frac{P(x, t)}{P_{0}},
$$

where $T(x, 0)=T_{w}+\left(T(0,0)-T_{w}\right) e^{(-\beta x)}$-initial destribution of temperatutre along pipeline; $T_{w}$-is temperature of pipeline's wall (soil), value of $\beta$-difined from the boundary conditions and has the following form:

$$
\beta=-\frac{1}{L} \ln \frac{\left(T(L, 0)-T_{w}\right)}{\left(T(0,0)-T_{w}\right)} .
$$

So explicit functions $T_{h d r}(x, t)=16.1 \lg \mathrm{P}(x, t)+173.6377$ and (32) gives possibility to define location of hydrates formation in main gas pipelines with different characteristics of pipeline and gas.

\subsection{Results of numerical calculation}

The length of the South Caucasus Pipeline (SCP) is $691 \mathrm{~km}$, with $443 \mathrm{~km}$ in Azerbaijan and $248 \mathrm{~km}$ in Georgia. The diameter is a 42 -inch $(1.0668 \mathrm{~m})$. Taking into account the SCP averaged technical parameters calculations were carried out by the following parameters: pipeline length $L=122 \mathrm{~km}$, pressure at $x=0$ (discharge pressure) $P_{0}=8.4 \mathrm{MPa}$, pressure at end point $P_{1}=7.88 \mathrm{MPa}$, temperature at $x=0$ (discharge temperature) $T_{0}=315.5 \mathrm{~K}$, temperature at $x=L$ (suction temperature) $T_{L}=286 \mathrm{~K}$, wall (soil) temperature $T_{w}=285 \mathrm{~K}$, specific heat at constant pressure $C_{p}=2278 \mathrm{~J} /(\mathrm{kgK})$, speed of sound $C=300 \mathrm{~m} / \mathrm{s}$. 
(a)

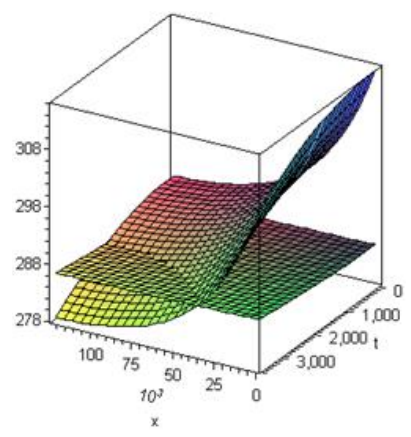

(b)

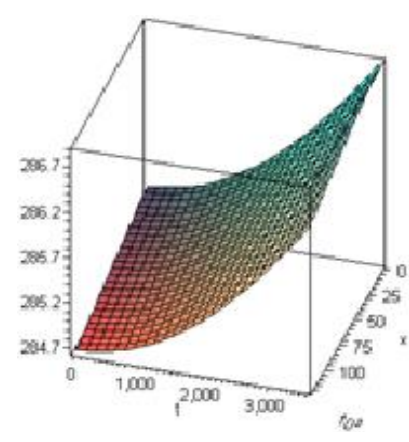

(c)

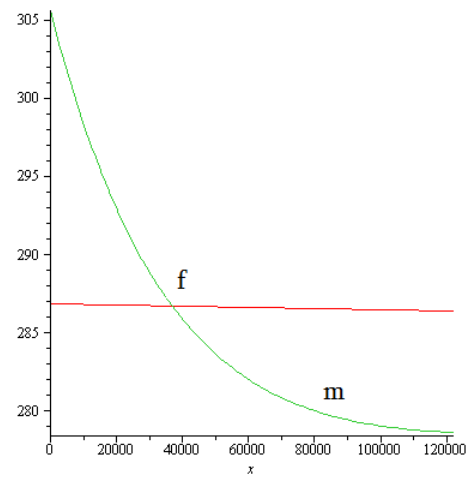

Fig.1. Temperature profiles along the pipeline during $t=3600 \mathrm{~s}$ (a) profiles (intersection) of $T(x, t)$ and $T_{\text {had }}(x, t)$ functions; (b) profile of $T_{\text {had }}(x, t)$ function; (c) temperatures distribution along the pipeline at $t=3600$ s.

Some results of numerical calculations executed by MAPLE software are presented in the Fig. 1. The Fig. 1 shows temperature profiles along the pipeline and possible section of hydrates formation in the main gas pipeline. Namely, in the Fig. 1a the horizontal plane is constructed on the basses of the equilibrium hydrates formation formula:

$$
T_{h d r}=s \lg P-u,
$$

and its special temporally distribution is depicted on the Fig. 1b. The lower part of the $T(x, t)$ function (below the intersection with $T_{h d r}$ ) are specifying a possible section of hydrates formation under condition of gas fully saturation by water vapor (Fig. 1a). It should be noted that generally gas is unsaturated by water vapor in the last section of pipeline (below the point $m$ ) as there is keeping almost the constant value of temperature, the gas pressure is coming down fast and so formation of hydrates is impossible (the beginning of this section is pointed by the point $f$ ). So a section of hydrate formation is the section $m f$ (Fig. 1c). So depending on the values and character of the gas temperature and pressure distribution along the main pipeline and on the values of initial moisture content (dew point of gas) perhaps it gives an opportunity for possibly formation of the several local hydrates shutters in the gas main pipeline.

\section{Conclusions}

At present, the process of climate change is evident in the South Caucasus region and especially in Georgia. Accelerated melting of glaciers, more frequent droughts and heavy rainfall are good indicators of current climate change in Georgia. The southeastern part of Georgia is especially sensitive to the catastrophic processes of drought and heavy rainfall where the main gas pipelines pass. At the same time, over the past four decades, the number of extreme weather events and natural disasters (floods, landslides, storms, downpours, hail) has significantly increased (approximately three times compared to the 60-year period of the last century) in Georgia. Thus, external factors of extreme temperature and pressure drop are of particular importance for the main gas pipelines of Georgia. In this context, the determination of the capabilities of models with respect to variables in the compilation of scenarios of extreme climate events is an urgent task.

Gas hydrates (ice like crystals) formation in gas conveyance pipelines prevents transporting of gas and not infrequently causes closes of the pipelines. So gas hydrates 
formation should be timely prevented. To stop gas hydrate formation in gas transporting pipelines, from existing methods the mathematical modelling with hydrodynamic method is more acceptable. In this study a new approach for the prediction of possible location of hydrate's origin in the main pipelines taking into consideration gas non-stationary flow is proposed. The dew point location is obtained with the help of finding the intersection of equilibrium temperature plot (of hydrate generation) and corresponding curve of gas temperature. For solving the problem the system of partial differential equations governing gas non-stationary flow in main gas pipeline is used. The problem solution for gas adiabatic flow is presented. For verification of the method a general test has been created. The results of calculations on the basis of observation data have shown that the performed simulations were much closer to the results of observation.

The research was funded by Shota Rustaveli National Scientific Foundation Grant No. FR17_548.

\section{References}

1. Bushkovsky, A. (1979). Characteristic system of distribution of parameters. Nauka Press.

2. De Read, J.R. (1987). Pipes and pipeline international, 32(1), 8.

3. Davitashvili, T. (2009). Natural disasters and surface and subsurface water pollution risk assessment for some regions of Georgia. NATO Science for Peace and Security Series C: Environmental Security, 83-89. https://doi.org/10.1007/978-90-481-2344-5 9

4. Gong, J., Shi, B., \& Zhao, J. (2010). Natural gas hydrate shell model in gas-slurry pipeline flow. Journal of Natural Gas Chemistry, 19(3), 261-266. https://doi.org/10.1016/s10039953(09)60062-1

5. Taylor, C.E., Kwan, J.T. (2004). Advances in the study of gas hydrates. London, United Kingdom: Kluwer Academic/Plenum Publishers Press.

6. Rice, W. (2006). Hydrogen production from methane hydrate with sequestering of carbon dioxide. International Journal of Hydrogen Energy, 31(14), 1955-1963. https://doi.org/10.1016/j.jijhydene.2006.01.017

7. Steinberg, M. (1989). Modern and prospective technologies for hydrogen production from fossil fuels. International Journal of Hydrogen Energy, 14(11), 797-820. https://doi.org/10.1016/03603199(89)90018-9

8. Ahsan, S. (2013). Transport by existing gas pipeline of gas mixture. International Journal of Chemistry, 1(15), 1-6.

9. Davitashvili, T., Gubelidze, G., \& Sharikadze, M. (2015). Reports of Enlarged Session of the Seminar of I. Vekua Institute of Applied Mathematics, 29(4), 34-37.

10. Environmental and Social Impact Assessment Baku - Tbilisi - Ceihan. (2012). Non-technical Executive Summary Project No: P8107, 1-312.

11. Davitashvili, T., Gubelidze, G., \& Samkharadze, I. (2011). Leak detection in oil and gas transmission pipelines. In book Informational and Communication Technologies - Theory and Practice. New York: Inprint Nova.

12. Zarinabadi, S., \& Samimi, A. (2011). Problems of hydrate formation in oil and gas pipes deals. Australian Journal of Basic and Applied Sciences, 5(12), 741-745.

13. Sloan, E.D. (2003). Fundamental principles and applications of natural gas hydrates. Nature, 426(6964), 353-359. https://doi.org/10.1038/nature02135

14. Yufin, V.A. (1978). Gas and oil pipeline transportation. Moscow, Russian Federation: Nedra Press.

15. Østergaard, K.K., Tohidi, B., Danesh, A., Todd, A.C., \& Burgass, R.W. (2000). Gas hydrate equilibria of 2,3-dimethylbutane and benzene with methane and nitrogen. Chemical Engineering Research and Design, 78(5), 731-737. https://doi.org/10.1205/026387600527914 
16. Tohidi, B., Danesh, A., Todd, A.C., Burgass, R.W., \& Østergaard, K.K. (1997). Equilibrium data and thermodynamic modelling of cyclopentane and neopentane hydrates. Fluid Phase Equilibria, 138(1-2), 241-250. https://doi.org/10.1016/s0378-3812(97)00164-7

17. Haghighi, H., Azarinezhad, R., Chapoy, A., Anderson, R., \& Tohidi, B. (2007). Hydraflow: Avoiding gas hydrate problems. EUROPEC/EAGE Conference and Exhibition. https://doi.org/10.2118/107335-ms

18. Østergaard, K.K., Masoudi, R., Tohidi, B., Danesh, A., \& Todd, A.C. (2005). A general correlation for predicting the suppression of hydrate dissociation temperature in the presence of thermodynamic inhibitors. Journal of Petroleum Science and Engineering, 48(1-2), 70-80. https://doi.org/10.1016/j.petrol.2005.04.002

19. Mohammadi, A.H., Tohidi, B., \& Burgass, R.W. (2003). Equilibrium data and thermodynamic modeling of nitrogen, oxygen, and air clathrate hydrates. Journal of Chemical \& Engineering Data, 48(3), 612-616. https://doi.org/10.1021/je025608x

20. Mohammadi, A.H., Chapoy, A., Tohidi, B., \& Richon, D. (2006). Gas solubility: A key to estimating the water content of natural gases. Industrial \& Engineering Chemistry Research, 45(13), 4825-4829. https://doi.org/10.1021/ie051337i

21. Avlonitis, D. (1994). A scheme for reducing experimental heat capacity data of gas hydrates. Industrial \& Engineering Chemistry Research, 33(12), 3247-3255. https://doi.org/10.1021/ie00036a046

22. Bobrovski, S.A. (1976). Gas pipeline transportation. Moscow, Russian Federation: Nauka Press.

23. Matko, D., Geiger, G., \& Gregoritza, W. (2000). Pipeline simulation techniques. Mathematics and Computers in Simulation, 52(3-4), 211-230. https://doi.org/10.1016/s0378-4754(00)00152-x

24. Tikhonov, A.N., \& Samarskii, A.A. (1977). Equations of mathematical physics. Moscow, Russian Federation: Nauka Press. 